\title{
Deletion of UNC5B in Kidney Epithelium Exacerbates Diabetic Nephropathy in Mice
}

\author{
Punithavathi Ranganathan $^{\mathrm{a}}$ Riyaz Mohamed $^{\mathrm{a}}$ Calpurnia Jayakumar ${ }^{\mathrm{a}}$ \\ Michael W. Brands ${ }^{b}$ Ganesan Ramesh ${ }^{a}$ \\ Departments of a Medicine and Vascular Biology Center and ${ }^{b}$ Physiology, Georgia Regents University, Augusta, Ga., USA
}

\author{
Key Words \\ UNC5B · Diabetic nephropathy $\cdot$ Netrin-1
}

\begin{abstract}
Background: Guidance cue netrin-1 was shown to have protective effects in diabetic nephropathy. However, the role of its receptor UNC5B in diabetic kidney disease is unknown. Moreover, whether netrin-1 is protective against diabetic kidney disease in a genetic model of nephropathy and in the nephropathy prone DBA background is also unknown. The aim of this study was to determine the significance of UNC5B in tubular epithelial cells in chronic kidney disease due to diabetes and evaluate whether netrin- 1 is also protective in the case of a nephropathy-prone mouse. Methods: Proximal tubular epithelium-specific UNC5B knockout mice as well as heterozygous UNC5B knockout mice were used to determine the roles of UNC5B in nephropathy. Diabetes was induced in these tissue-specific knockout, heterozygous and WT mice, and albuminuria was then monitored. Results: WT and heterozygous diabetic mice developed significant albuminuria at 8 weeks after induction of diabetes as compared to buffer-treated control mice. However, albuminuria was significantly more pronounced in mice with proximal tubule specific deletion of UNC5B. Transgenic overexpression of netrin-1 in proximal tubules in the DBA background and administration of recombinant netrin-1 to Ins2Akita mice also significantly reduced diabetes-induced albuminuria and suppressed glomerular and interstitial lesions.
\end{abstract}

Conclusion: Our data suggested that netrin-1 signaling in proximal tubular epithelium may play a critical role in the protection of kidney against diabetic kidney disease.

(c) 2015 S. Karger AG, Basel

\section{Introduction}

Diabetic nephropathy is a leading cause of end-stage renal disease (ESRD) and a major source of morbidity, mortality and health care expenditures. In the United States, approximately 20.8 million people, or $7.0 \%$ of the population, are estimated to have diabetes, and the incidence is growing. Diabetes has become the primary cause of ESRD in the United States, and the incidence of type 2 diabetes mellitus continues to grow worldwide. Approximately, $44 \%$ of new patients entering dialysis in the United States are diabetics $[1,2]$. Early diagnosis of diabetes and intervention are critical in diminishing the progression to renal failure seen in many type 1 and a significant percentage of type 2 diabetics. Currently, no test is available to predict the development of diabetic nephropathy. Moreover, therapies for diabetic nephropathy, mainly glycemic and blood pressure control, provide incomplete protection against renal disease progression [3]. There is a pressing need to identify the underlying patho-

P.R. and R.M. equally contributed to this work.

\section{KARGER 125}

(c) 2015 S. Karger AG, Base

$0250-8095 / 15 / 0413-0220 \$ 39.50 / 0$

E-Mail karger@karger.com

www.karger.com/ajn
Ganesan Ramesh, $\mathrm{PhD}$

Department of Medicine and Vascular Biology Center, CB-3702

Georgia Regents University, 1459 Laney-Walker Blvd

Augusta, GA 30912 (USA)

E-Mail gramesh@gru.edu 
genic mechanisms of diabetic nephropathy in order to develop targeted therapeutic agents with enhanced efficacy. Recent studies suggest that diabetic nephropathy is an inflammatory disease, and inflammatory mediators such as cytokines and chemokines play major roles in disease development and progression $[4,5]$. Recent studies suggest the presence of endogenous pathways, such as netrin-1, that favorably regulate inflammation in diabetic nephropathy [6]. Deregulation of this protective pathway may accelerate disease development in the chronic diabetic milieu.

Netrin-1 is a laminin-related secreted molecule that has been identified as a neuronal guidance cue, directing neurons and their axons to targets during development of the nervous system. However, netrins are widely expressed outside the nervous system, including vascular endothelial $[7,8]$ and kidney tubular epithelial cells [8], suggesting a broader role in regulating pathophysiology. Netrin-1 expression in vascular endothelial cells forms a critical barrier for leukocyte migration, and downregulation of netrin-1 exacerbates inflammation in acute models of organ injury $[7,8]$. Netrin-1 mediates its biological activity by binding to two families of vertebrate receptors: the deleted in colorectal cancer (DCC) family and the uncoordinated-5 homolog (UNC5) family [9]. Several studies strongly suggest that the UNC5B receptor mediates the protective function of netrin-1 during acute kidney injury $[10,11]$. Moreover, significant expression of UNC5B, but none of the other netrin receptors, was found in immune cells, endothelial cells and proximal renal tubular epithelial cells $[10,12]$. Netrin-1 overexpression [6] or administration of recombinant netrin-1 [13] to a model of streptozotocin (STZ)-induced type 1 diabetic mice protected the kidney from inflammation, albuminuria and fibrosis. However, the receptor that mediates netrin-1 activity is unknown. Moreover, whether netrin-1 overexpression or administration of recombinant netrin-1 to a genetic model of type 1 diabetic mice and other nephropathy prone mice, such as the DBA strain, are also protective is unknown. The current study was carried out to answer these questions.

\section{Material and Methods}

\section{Ethical Statement}

All animal handling and procedures were performed in accordance with protocols for these studies that have been approved by the Institutional Animal Care and Use Committee at Georgia Regents University (Protocol \#2011-0348). All experiments in animals were performed aseptically and every attempt was made to minimize pain and discomfort.

UNC5B and Diabetic Nephropathy
Mouse Strains

The generation of the $U N C 5 B$-flox alleles has been described previously $[11,14,15]$. GGT-cre mice were generously provided by Eric Nelson at Vanderbilt University School of Medicine. Genotypes were determined by PCR analysis of genomic DNA isolated from tail clips; amplification primers and conditions used for UNC5B mice were: $5^{\prime}$-TAGCCTCAGGGTCTACTGTCTG-3'; $5^{\prime}$-CTCTCAGACTTCTCAAAGAGATTC- $3^{\prime}$; and $5^{\prime}$-CCACTG TATGCCAGACGACATG- $3^{\prime}$ under conditions of $94^{\circ} \mathrm{C}$ for $30 \mathrm{~s}$, $62^{\circ} \mathrm{C}$ for $20 \mathrm{~s}$ and $72^{\circ} \mathrm{C}$ for $40 \mathrm{~s}$. GGT-cre mice were genotyped using the primers: $5^{\prime}$-AGGTGTAGAGAAGGCACTTAGC- $3^{\prime}$; and $5^{\prime}$-CTAATCGCCATCTTCCAGCAGG- $3^{\prime}$ under conditions of $94^{\circ} \mathrm{C}$ for $30 \mathrm{~s}, 58^{\circ} \mathrm{C}$ for $20 \mathrm{~s}$ and $72^{\circ} \mathrm{C}$ for $40 \mathrm{~s}$. The Institutional Animal Care and Use Committee of Georgia Regents University approved all of the protocols and procedures for using animals (approval number BR10-10-369).

Proximal Tubular Epithelial Cell-Specific Deletion of UNC5B

Creation and characterization of the $\mathrm{UNC} \mathrm{B}^{\text {flox }}$ allele were described previously $[14,15]$. To systematically inactivate UNC5B in a tissue-specific fashion, animals homozygous for the conditional allele (UNC5 $\mathrm{B}^{\text {flox/flox }}$ ) were mated with animals heterozygous for the $U N C 5 B$ null allele $\left(\mathrm{UNC} \mathrm{B}^{+/-}\right)$. The $\mathrm{UNC} \mathrm{B}^{\text {flox/- }}$ progeny were mated with mice carrying the Cre gene under the control of gamma-glutamyl transferase (GGT) promoter whose activity was restricted to proximal tubular epithelial cells. The tubular-restricted GGT promoter was shown to be active only in adulthood but not during embryo development [16]. Deletion was confirmed by PCR and immunohistochemical staining of UNC5B in kidney sections (fig. 1) as previously described [11]. Deletion of $U N C 5 B$ in proximal tubular epithelial cells resulted in mice that survived to adulthood and that appeared to be normal.

\section{STZ-Induced Diabetes}

The diabetes induction protocol followed for mice was described by the Animal Models of Diabetic Complication Consortium (AMDCC) (http://www.diacomp.org/shared/protocols. aspx) [17-19]. Eight-week-old UNC5B flox/-/GGT-cre mice and littermates without cre $\left(\mathrm{UNC} 5 \mathrm{~B}^{\text {flox/- }}\right.$ ) or wild-type $\left(\mathrm{UNC} 5 \mathrm{~B}^{\text {flox/flox }}\right.$ ) mice were given streptozotocin (STZ) $(50 \mathrm{mg} / \mathrm{kg}$ body weight (BW) in citrate buffer) in multiple doses (every $24 \mathrm{~h}$, total of five doses). Blood glucose and urine albumin were measured at 1 week after STZ injection and every four weeks thereafter. Relion glucose strips with Relion glucose monitor were used to measure the blood glucose in mice. About 6-10 animals/groups were used in all experiments.

Netrin-1 Transgenic Mice Backcrossed to DBA/2J

The DBA mouse strain is shown to have accelerated development of nephropathy and shows robust proteinuria $[18,19]$. Therefore, we crossed transgenic mice that overexpress netrin-1 in proximal tubular epithelium under control of the L-fatty acid binding protein promoter $[6,20]$ with DBA/2J (Jackson Laboratories) mice. Six-week-old mice from the first generation (F1) that are positive and negative for the netrin-1 transgene were made diabetic using low-dose STZ (50 mg/kg BW/day for 5 days). Kidney function and proteinuria were monitored for another 8 weeks after the confirmation of diabetes. Urine was collected over a $24 \mathrm{~h}$ period and animals were sacrificed. Kidney tissues were processed for histopathological analysis. 


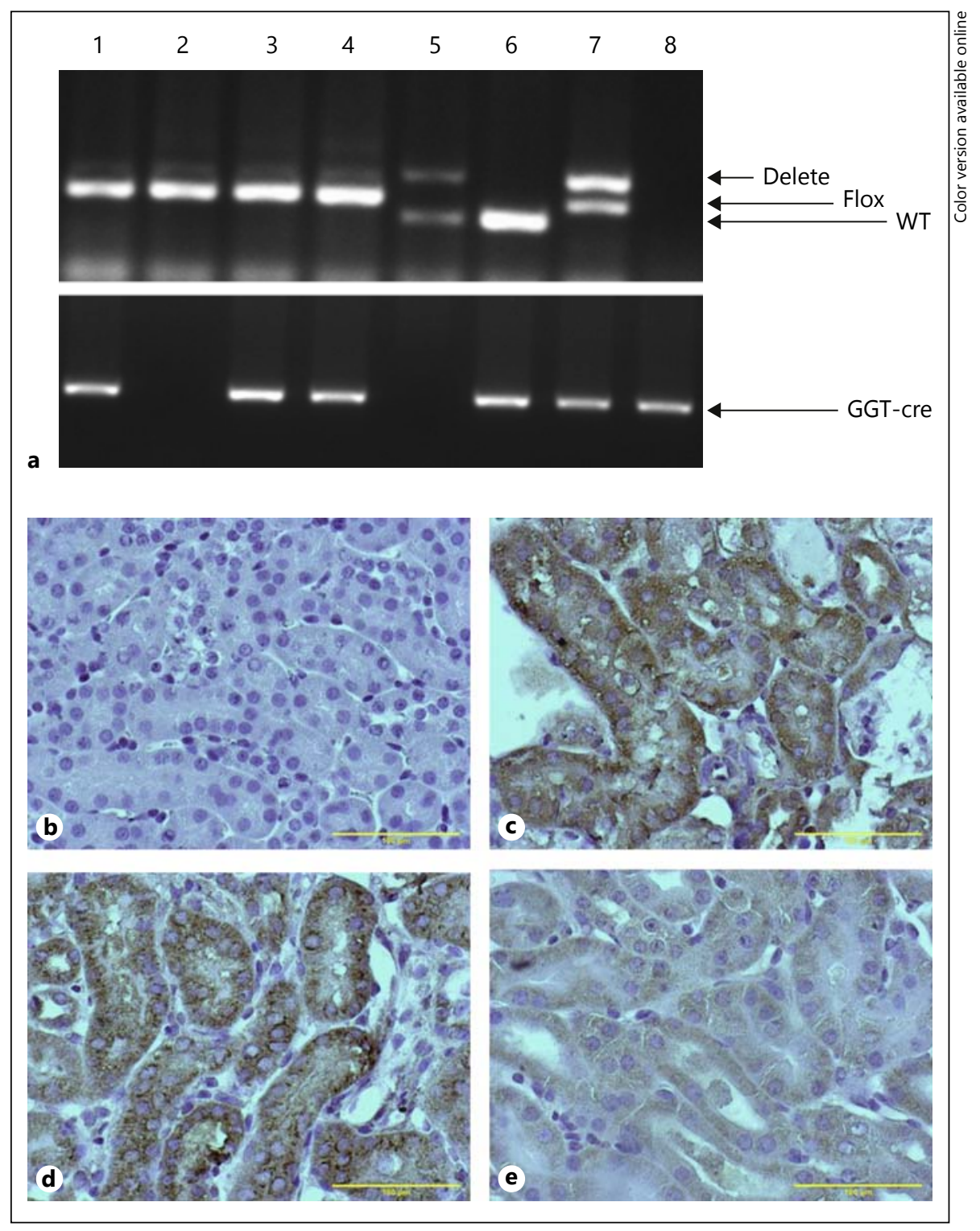

Fig. 1. Characterization of kidney proximal tubular epithelial-specific deletion of UNC5B. a Genomic PCR showing successful generation of different genotypes, UNC5B $\mathrm{B}^{\mathrm{WT} /-/ \text { GGT-cre }}$ and UNC5B $\mathrm{B}^{\text {flox/-/GGT-cre }}$. The band marked deleted represents whole body deletion of a single allele for UNC5B. Immunohistochemical localization of $\mathrm{UNC5B}$ receptor in $\mathrm{UNC}^{\mathrm{B}} \mathrm{B}^{\text {flox/flox }}(\mathbf{c})$, $\mathrm{UNC}^{\text {flox/- }}(\mathbf{d})$ and UNC5B $\mathrm{B}^{\text {flox/-/GGT-cre }}(\mathbf{e})$ mice. Antibody control is shown in b. Flox/ flox without cre and heterzygous knockout mice without cre showed UNC5B staining in the proximal tubular epithelial cells, whereas GGT-cre positive mice showed no staining for UNC5B, suggesting successful deletion of the floxed UNC5B gene. Scale bar: $100 \mu \mathrm{M}$.

Recombinant Netrin-1 Administration to Ins2Akita Mice

Male 12-week-old DBA/2J mice (wild-type or with the Akita mutation (JAX\# 007562, D2.B6-Ins2Akita/MatbJ) were purchased from Jackson Laboratories. Mice with the Akita mutation developed diabetes when they are 4 weeks old. At the age of 12 weeks, Akita mice were treated with recombinant human netrin-1 $(5 \mu \mathrm{g} /$ animal/every $48 \mathrm{~h}$, intraperitoneally) or vehicle ( $0.1 \% \mathrm{BSA})$ for a period of 6 weeks. $24 \mathrm{~h}$ urine was collected for determining the albumin excretion rate. Animals were sacrificed to collect plasma and kidney tissues, which were fresh-frozen for analysis of gene expression or histopathological analysis.

\section{Measurement of Urine Albumin Excretion Rate (AER)}

Excretion of urinary albumin was determined using the albumin-to-creatinine ratio (ACR) in $24 \mathrm{~h}$ urine collections. The $24 \mathrm{~h}$ urine was collected using the Nalgene ${ }^{\circledR}$ Metabolic Cage System
(Rochester, N.Y., USA), which is designed to allow efficient separation of urine and feces from a single mouse. The concentration of albumin in urine was quantified using an ELISA kits (Bethyl Laboratories, Inc., Montgomery, Tex., USA), and urine creatinine was determined by using a creatinine assay kit (Diazyme Laboratories, Poway, Calif., USA).

\section{Cytokine and Chemokine Measurements}

Cytokines and chemokines in serum and urine were measured using an ELISA array kit from eBioscience.

\section{Histological Analysis}

Kidney tissue was fixed in buffered $10 \%$ formalin for $12 \mathrm{~h}$ and then embedded in paraffin wax. For assessment of injury, $5 \mu \mathrm{M}$ sections were stained with periodic acid-Schiff (PAS) followed by hematoxylin. To assess fibrosis in the kidney, kidney tissue sec- 
tions were stained with Trichrome. Stained sections were photographed using an Olympus inverted microscope with a color CCD camera.

\section{Glomerular Morphometry}

At the time of sacrifice, kidneys were harvested for pathological examination. One kidney was fresh-frozen for gene expression analysis, and the other was fixed in $10 \%$ neutral-buffered formalin (Sigma-Aldrich Co., St. Louis, Mo., USA). The formalin-fixed tissue was embedded in paraffin, and $4-\mu \mathrm{m}$ sections were stained with periodic acid-Schiff stain (American Histolabs, Gaithersburg, Md., USA). Glomerular sclerosis was quantified using a semi-automatic image analysis technique with an examination of the total cortical area. To perform morphologic analyses, a total of 30 glomeruli were randomly selected from each kidney by moving the slide from the outer to the inner cortex in a random fashion to obtain non-overlapping sample fields. Glomerular images were recorded using a CCD camera (Olympus DP72 color CCD camera) (Olympus USA, Pittsburg, Pa., USA) mounted on an Olympus light microscope. The glomerular tuft was traced, and the enclosed area was calculated with Cellsens Standard software.

For quantification of mesangial extracellular matrix, 3- $\mu \mathrm{m} \mathrm{sec-}$ tions from paraformaldehyde-fixed, paraffin-embedded kidney slices were stained using PAS. Mesangial area was expressed quantitatively by calculating the percentage of the total glomerular area that was PAS positive and nucleus free area [21, 22]. Fifteen glomerular tufts per animal were chosen randomly for analysis.

Statistical Analysis

Data are presented as the mean \pm SEM. $\mathrm{p}<0.05$ was considered significant. Statistical analyses were performed using Graphpad Instat 3 (GraphPad Software, San Diego, Calif., USA). All assays were performed in duplicate or triplicate. Statistical significance was assessed by an unpaired, two-tailed Student t-test for single comparison or ANOVA for multiple comparisons.

\section{Results}

\section{Proximal Tubular Epithelial Cell-Specific Deletion}

of UNC5B in Mice Exacerbate Diabetes-Induced

\section{Albuminuria}

To determine the role of the tubular epithelial UNC5B receptor in chronic kidney disease, such as diabetic nephropathy, mice with the UNC5B $\mathrm{B}^{\text {flox/-/GGT-cre }}$ allele and littermates without cre $\left(\mathrm{UNC} 5 \mathrm{~B}^{\text {flox/- }}\right.$ ) or wild-type were made diabetic as described in Materials and Methods. Buffer-treated, non-diabetic control animals did not show any increase in blood glucose level or increase in albuminuria (fig. 2a-c). However, diabetic wild-type mice and heterozygous knockout mice (UNC5B ${ }^{\text {flox/-}}$ ) showed a significant increase in albuminuria as compared to non-diabetic controls. Interestingly, the increase in albuminuria was much more pronounced in UNC5B $\mathrm{B}^{\text {flox/-/GGT-cre mice }}$ (fig. 2a and $b$ ), suggesting that UNC5B signaling in proximal tubular epithelium may play an important role in controlling diabetes-induced albuminuria. Blood glucose level and kidney hypertrophy were increased significantly in all three groups over controls, but no differences were seen among the diabetic groups. Consistent with albuminuria, PAS-stained sections showed expansion of the mesangial matrix and deposition of proteinaceous materials in the glomerulus in diabetic animals, which was more

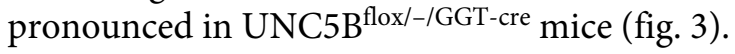

\section{Effects of Netrin-1 Overexpression in Tubular}

Epithelium in the Nephropathy Prone DBA Mouse

\section{Background on Albuminuria}

Previous studies had shown that netrin-1 overexpression in proximal tubular epithelium is protective against diabetic nephropathy in the CB57BL/6 background strain, which showed a mild form of nephropathy [6]. To determine whether netrin-1 offers protection against diabetes-induced albuminuria in the nephropathy-prone DBA mouse background, we performed an F1 backcross as described in Materials and Methods. As shown in figure 4, no difference was observed between wild-type (WT) and netrin-1 transgenic mice for blood glucose level (fig. 4a) and kidney weight/body weight ratio (fig. 4b) after induction of diabetes. However, wild-type mice showed a significant increase in albuminuria over control. These changes were significantly reduced in netrin- 1 transgenic diabetic animals (fig. $4 \mathrm{~d}$ ), suggesting netrin-1 is protective even in the nephropathy-prone mouse background. Consistent with albuminuria, the PASstained sections showed an increase in glomerular area (fig. 4f) and mesangial expansion (fig. $4 \mathrm{e}$ and g) in wildtype kidney but not in the netrin-1 transgenic kidney.

Inflammation is a mediator of diabetic nephropathy. Previous studies had demonstrated that netrin-1 regulates inflammation in diabetes [6]. Consistent with previous studies, the excretion of IL- 6 and TNFa was significantly increased in diabetic mice (fig. $5 \mathrm{a}$ and b). However, excretion of these cytokines was significantly lower in netrin-1 transgenic diabetic mice (Tg3-DBA mice) as compared to wild-type diabetic mice. To determine whether kidney specific overexpression of netrin-1 has any influence on systemic inflammation, IL- 6 and TNFa in serum was quantified. IL-6 level was significantly elevated in WT with diabetes. However, overexpression of netrin-1 in the kidney did not alter serum IL-6 levels (fig. 5c). TNFa level in serum was not increased significantly in both WT and netrin-1 transgenic diabetic mice over control mice (fig. 5d). These data suggest that kidney epithelium specific overexpression of netrin-1 does not alter systemic inflammation in diabetes. 


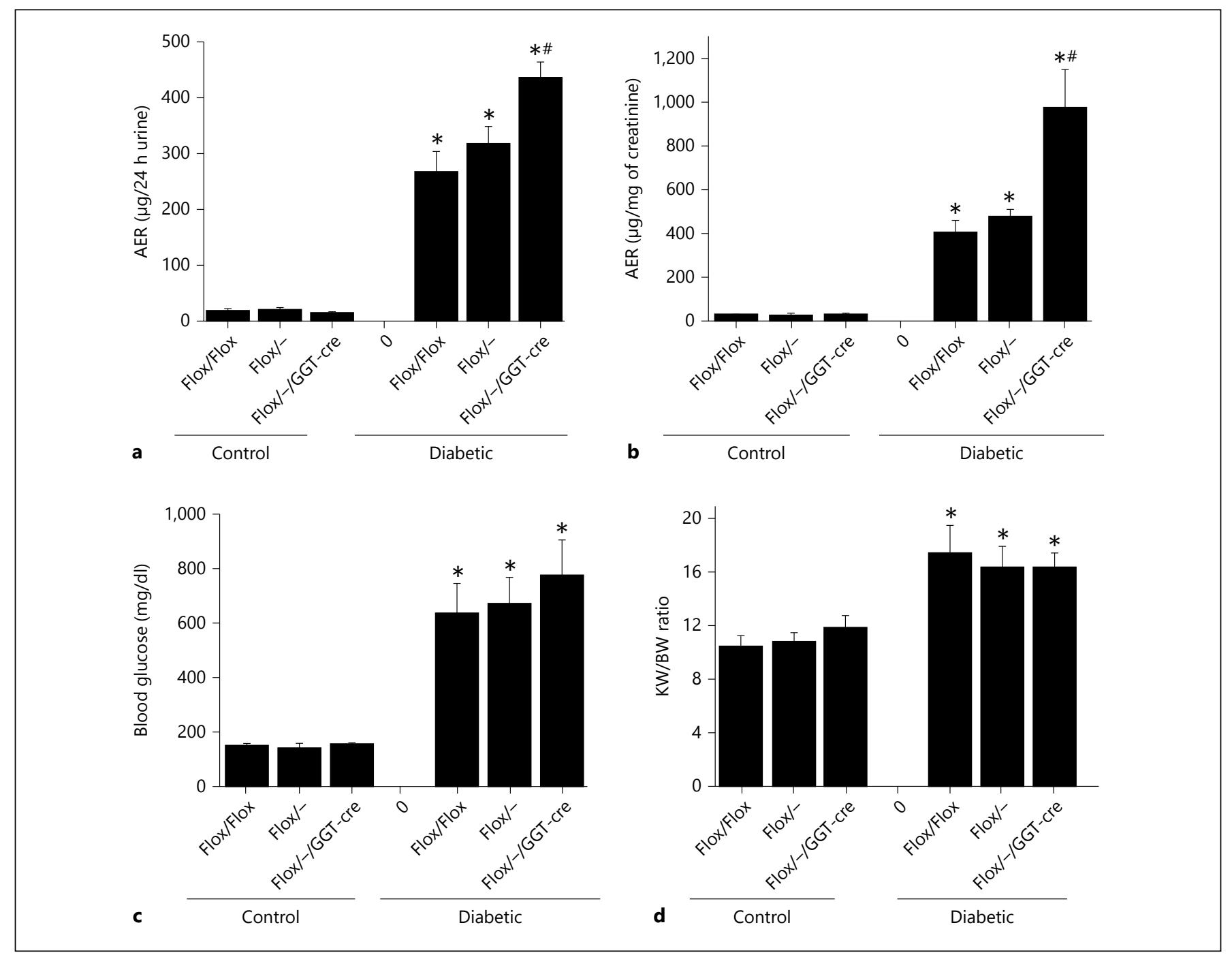

Fig. 2. Proximal tubule-specific deletion of UNC5B exacerbates diabetes-induced albuminuria. Mice with whole body, partial (one allele) (flox/-), or total (flox/-/GGT-cre) deletion of UNC5B in the proximal tubules, or their wild type (flox/flox) controls, were made diabetic as described in Materials and Methods. Eight weeks after induction of diabetes, albuminuria and kidney hypertrophy changes were assessed. a, b Diabetes-induced albuminuria expressed as albumin excretion rate (AER). Significant increase of AER in diabetic mice was seen as compared to non-diabetic con-

\section{Effect of Recombinant Netrin-1 Administration on}

\section{Albuminuria in Ins2Akita Diabetic Mice}

To translate our finding, we administered recombinant netrin- 1 to the most widely used type 1 diabetic mouse model for nephropathy as described in Materials and Methods. As shown in figure 6a, vehicle treated Ins2Akita mice showed a large increase in albumin excretion rate as compared to the wild-type control. Ad-

trols. Flox/-/GGT-cre mice showed exacerbated albuminuria as compared to diabetic WT and heterozygous UNC5B KO (flox/-) mice. ${ }^{*} \mathrm{p}<0.01$ versus control. ${ }^{*} \mathrm{p}<0.05$ versus other diabetic groups. $n=6-8$. c Blood glucose levels were equally elevated in all three groups of diabetic mice. ${ }^{*} \mathrm{p}<0.001$ versus control. d Kidney hypertrophy is significantly increased in all three groups, and UNC5B deletion did not alter the diabetes-induced kidney hypertrophy. ${ }^{*} \mathrm{p}<0.05$ versus control.

ministration of netrin-1 significantly suppressed albumin excretion as compared to vehicle-treated animals. Blood glucose (fig. 6b) and urine glucose levels (fig. 6c) were elevated in both vehicle and netrin-1 treated Ins2Akita mice as compared to wild-type control. However, the diabetes-induced increased kidney hypertrophy was significantly reduced with netrin-1 treatment. Consistent with the suppression of albumin excretion 
Fig. 3. Proximal tubule-specific deletion of UNC5B exacerbates diabetes-induced glomerular changes. Mice with whole body, partial (one allele) (flox/-), or total (flox/-/ GGT-cre) deletion of UNC5B in the proximal tubules, or their WT (flox/flox) controls, were made diabetic as described in Materials and Methods. Eight weeks after induction of diabetes, glomerular changes were assessed in PAS stained kidney sections. Scale bar: $100 \mu \mathrm{M}$. a PAS-stained kidney sections from diabetic and control mice showing enhanced accumulation of proteinaceous material and mesangial expansion in diabetic flox/- and flox/-/GGTcre mice compared to flox/flox controls. b Quantification of mesangial index. ${ }^{*} \mathrm{p}<$ 0.01 versus control. ${ }^{\#} \mathrm{p}<0.05$ versus flox/ flox (WT) diabetic. $\mathrm{n}=6-8$.

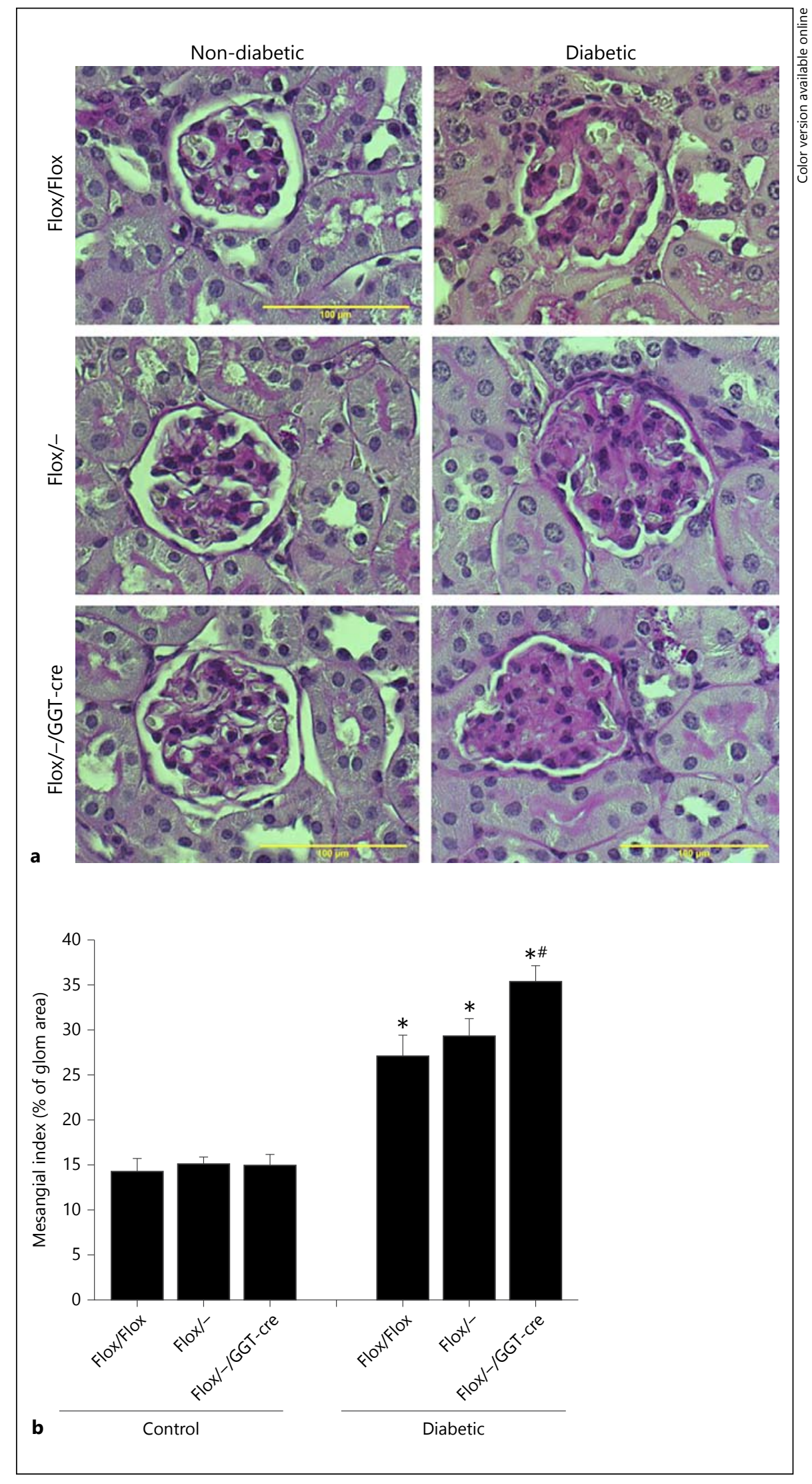




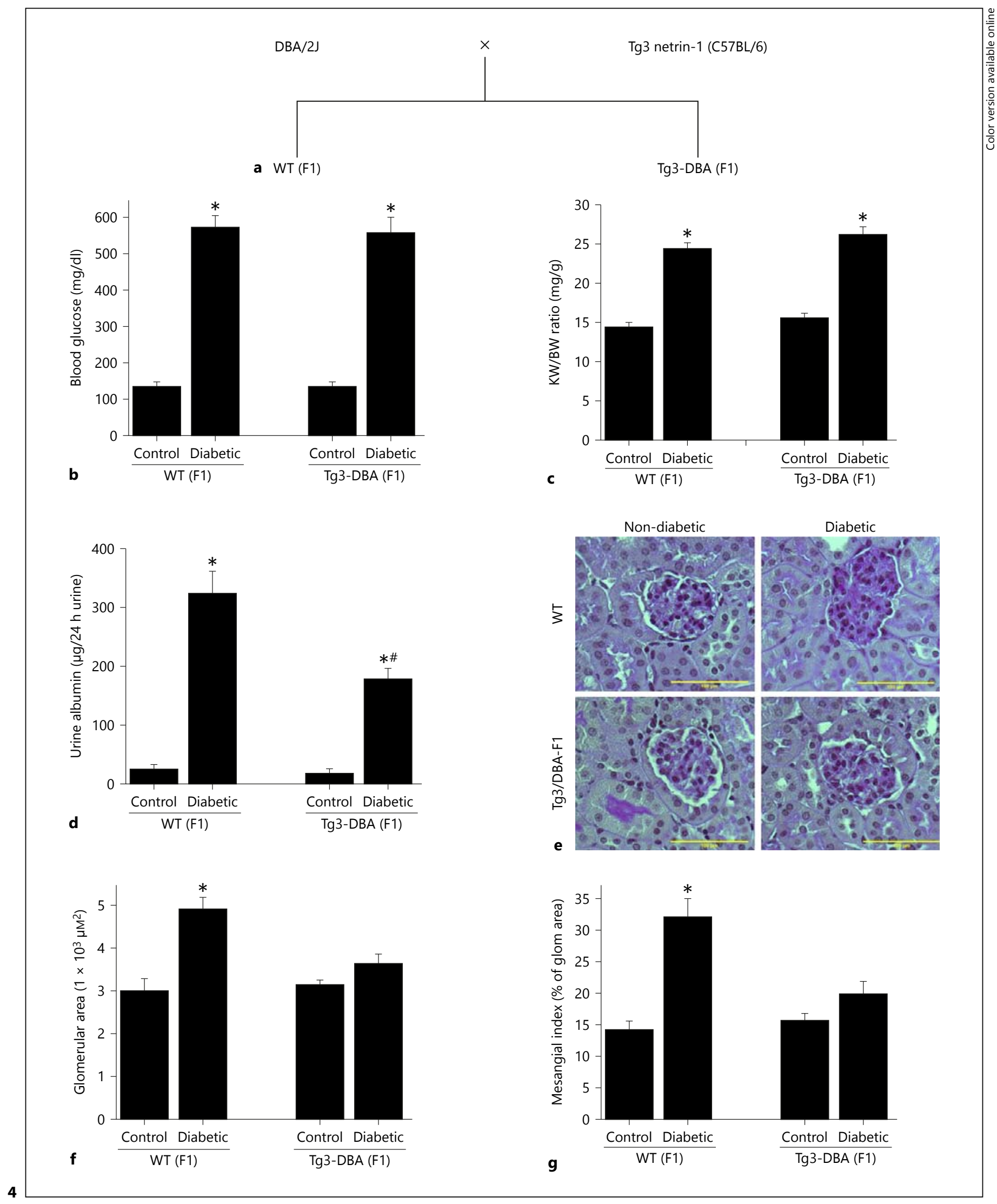

(For legend see next page.) 

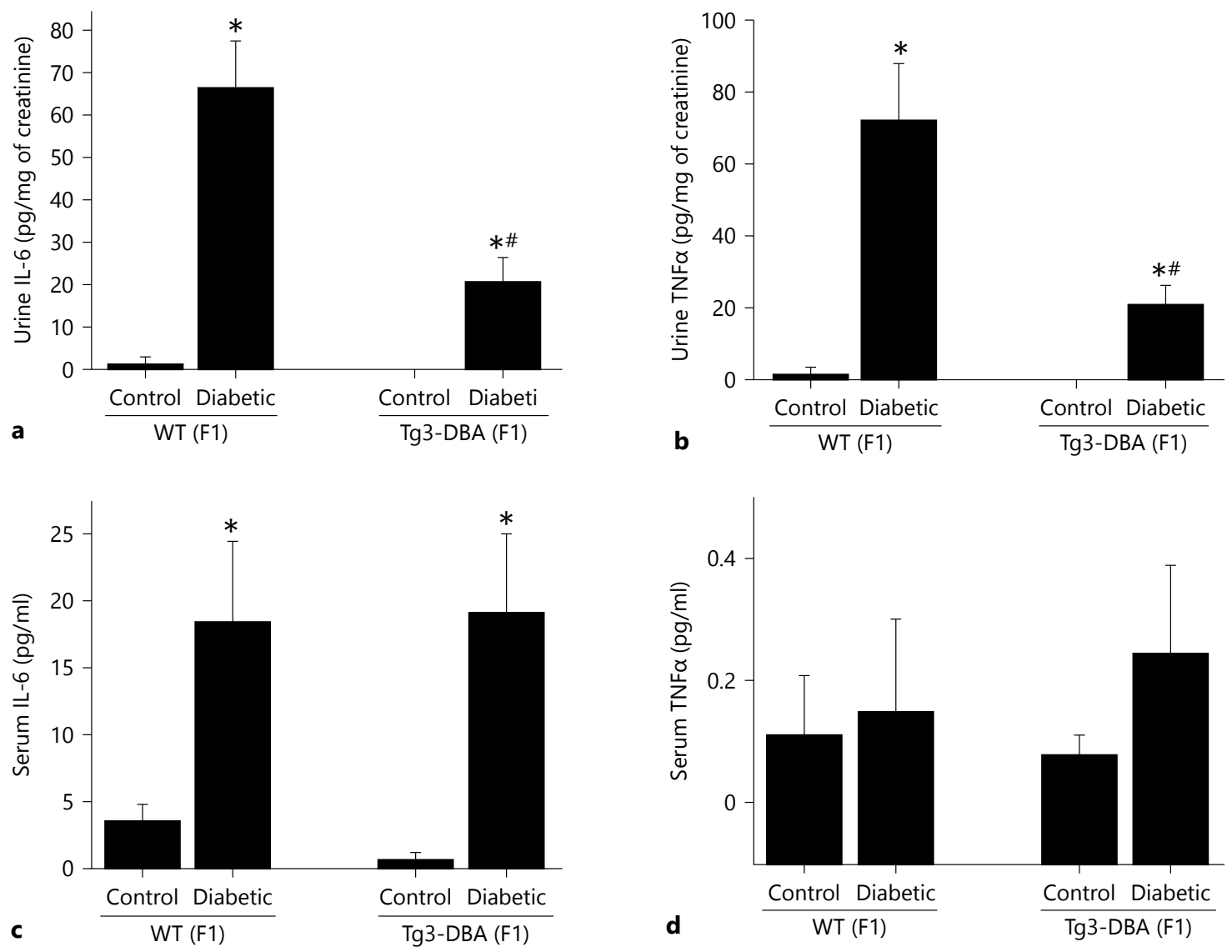

Fig. 5. DBA/netrin-1 transgenic (Tg3) diabetic mice excrete low levels of pro-inflammatory cytokines. F1 mice expressing the netrin-1 transgene and WT mice were made diabetic by low-dose STZ administration. IL-6 and TNFa excretion in urine were measured 8 weeks after the induction of diabetes and expressed as pg/ mg of urine creatinine. a Diabetes-induced increase in IL- 6 excretion in WT mice was significantly suppressed in Tg3-DBA mice.
${ }^{*} \mathrm{p}<0.01$ versus controls. ${ }^{\#} \mathrm{p}<0.001$ versus WT diabetic mice. b Diabetes-induced increase in TNFa excretion in WT mice was significantly suppressed in Tg3-DBA mice. ${ }^{*} \mathrm{p}<0.01$ versus controls. ${ }^{\#} \mathrm{p}<0.001$ versus WT diabetic mice. c Diabetes-induced increase in serum IL-6 levels in both WT and Tg3-DBA mice significantly. ${ }^{*} \mathrm{p}<0.05$ versus controls. d Diabetes does not alter serum TNFa levels in both WT and Tg3-DBA mice. $n=6-10$.
Fig. 4. $\mathrm{DBA} /$ netrin-1 transgenic ( $\mathrm{Tg} 3$ ) mice are resistant to diabetes-induced proteinuria and glomerulosclerosis. F1 mice expressing the netrin-1 transgene and WT mice were made diabetic by low dose STZ administration. Albuminuria and histological changes were measured 8 weeks after the induction of diabetes. a Breeding scheme of DBA/2J mice with netrin-1 transgenic mice. b Blood glucose level is equally elevated in wild-type and netrin-1 transgenic diabetic mice as compared to control. ${ }^{*} \mathrm{p}<0.001$ versus control. $n=8-10$. c Kidney weight/body weight ratio is equally increased in diabetic wild-type (WT) and transgenic mice as compared to controls. ${ }^{*} \mathrm{p}<0.001$ versus control. $\mathrm{n}=8-10$. $\mathbf{d}$ Diabetes-induced albuminuria was significantly suppressed in netrin-1 transgenic animals. ${ }^{*} \mathrm{p}<0.001$ versus control. ${ }^{\#} \mathrm{p}<0.05$ versus wild-type diabetic mice. $\mathrm{n}=8-10$. e Diabetes-induced mesangial expansion, accumulation of proteinaceous casts and basement membrane thickening were markedly reduced in netrin-1 transgenic mice. $\mathbf{f}$ Quantification of glomerular area. ${ }^{*} \mathrm{p}<0.05$ versus other groups. g Quantification of mesangial index. ${ }^{*} \mathrm{p}<$ 0.05 versus other groups. $n=6-8$. Scale bar: $100 \mu \mathrm{M}$. 


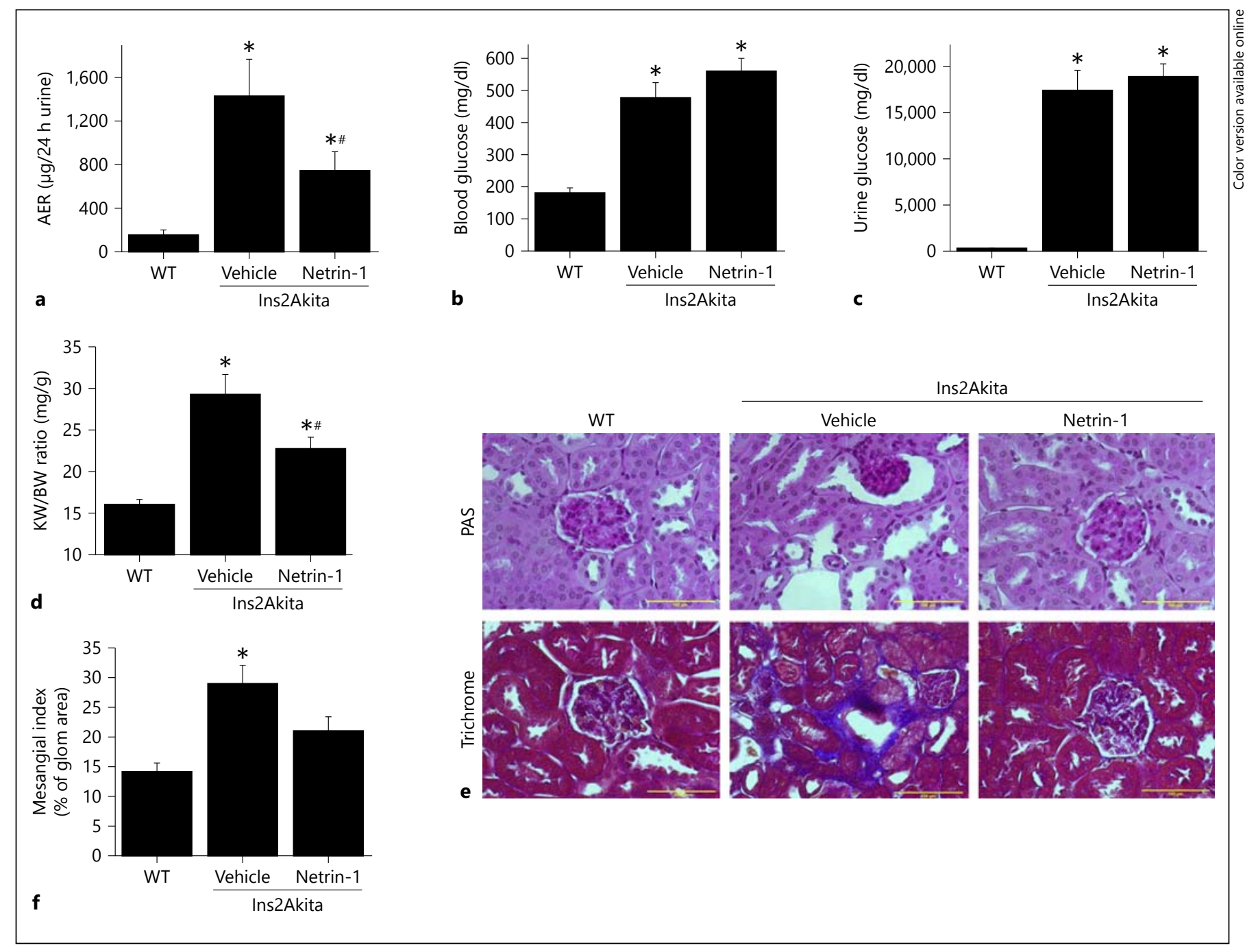

Fig. 6. Effects of recombinant netrin-1 administration on diabetesinduced albuminuria and glomerulosclerosis. Twelve-week-old wild-type (WT) or Ins2Akita mice were treated with recombinant netrin-1 ( $5 \mu \mathrm{g} /$ animal, every $48 \mathrm{~h}$ ) for 6 weeks. Animals were sacrificed at 18 weeks of age, kidney tissues were processed for histology, and proteinuria was quantified. Administration of recombi- nant netrin-1 reduces albuminuria (a), kidney hypertrophy (c) and glomerular sclerosis and interstitial fibrosis (e) but not the blood glucose level (b) or urine glucose excretion (d) in Ins2Akita mice. ${ }^{*} \mathrm{p}<0.001$ versus WT. ${ }^{\#} \mathrm{p}<0.01$ versus vehicle treated Ins 2 Akita mice. $\mathbf{f}$ Quantification of mesangial index. ${ }^{*} \mathrm{p}<0.05$ versus other groups. $\mathrm{n}=6-8$. Scale bar: $100 \mu \mathrm{M}$. with netrin-1 treatment, both tubular and glomerular alterations such as fibrosis, dilation of tubules and mesangial expansion were suppressed with netrin-1 treatment (fig. 6e and f).

\section{Discussion}

Endogenous protective pathways that counteract to suppress excess inflammation and cellular injury exist or are induced during acute or chronic disease conditions.
One such pathway is netrin- 1 and its receptor. This protective pathway was shown to play an important role in many acute and chronic diseases [6, 10, 20, 23-26]. Currently available therapeutics such as blood pressure control and intense glucose control are not able to reverse the disease process once diabetic nephropathy is established. Efforts to develop new therapies have been hindered by the lack of complete knowledge on protective pathways such as netrin-1 and UNC5B. In this study, we combined genetic and pharmacologic approaches to investigate the functional role of the neuronal guidance molecule ne- 
trin-1 and its receptor during diabetic nephropathy. Our findings suggest that renal epithelial cell specific deletion of the netrin-1 receptor UNC5B caused a far more severe course of kidney disease during STZ-induced diabetes mellitus as compared to diabetic littermate wild-type and heterozygous knockout (UNC5B-/+) mice. Overexpression of netrin-1 in the nephropathy prone mouse strain (DBA/2J) causes significant suppression of disease severity. Finally, recombinant netrin-1 administration to a genetic model of diabetic nephropathy (Ins2Akita) revealed robust treatment effects as shown by attenuated disease severity in all parameters that we assessed. Taken together, these studies indicate, for the first time, a protective role of UNC5B receptor signaling during diabetic nephropathy.

Previous studies from laboratory had suggested that UNC5B deletion in proximal tubular epithelium exacerbated acute kidney injury by increasing epithelial cell apoptosis and inflammation [11]. Consistent with these findings, the present study demonstrates that UNC5B signaling is critical even for chronic kidney disease. Two recent studies also demonstrated protective effects of netrin-1 using two different approaches. One study used overexpression in tubular epithelium [6], and the other study used heterozygous knockout mice and recombinant netrin-1 administration [13]. Both studies employed an STZ-induced mild form of nephropathy in a C57BL/6 background strain. It was demonstrated that netrin-1 suppresses albuminuria, in part, through the suppression of COX-2 expression and suppresses inflammation through the inhibition of $\mathrm{NF \kappa B}$ activation [6]. Moreover, netrin-1 also increased albumin uptake by epithelial cells. Therefore, it is possible that netrin-1 may suppress nephropathy through UNC5B by suppressing tubular epithelial damage and enhanced clearance of albumin from the lumen. This will reduce epithelial stress and inflammation, which will impact on podocyte function. Previ- ous studies, however, examined nephropathy in C57BL/6 mouse background, which is known to have a mild form of nephropathy and proteinuria $[18,19,27]$. It was not clear whether netrin-1 can be protective in a more nephropathy-prone mouse background such as DBA and Ins2Akita. Data presented in this manuscript suggest that netrin-1 is capable of protecting the kidney against diabetes-induced albuminuria and glomerulosclerosis even in a more nephropathy-prone mouse background. Moreover, netrin-1 may regulate inflammatory cytokine production, thereby reducing the development of nephropathy.

In conclusion, this study on the role of netrin-1 signaling during diabetic nephropathy demonstrated a protective role of the endogenous netrin-1 receptor UNC5B in attenuating disease severity. Moreover, tubular epithelial cell-derived netrin-1 may play a critical role in attenuating disease severity. Our study also demonstrated that the administration of netrin-1 could be therapeutic against diabetic nephropathy.

\section{Acknowledgments}

G.R. is supported by an R01 grant (1R01DK083379 - 01A5) from NIH-NIDDK. P.R. and R.M. are supported by postdoctoral fellowships from American Heart Association. The funders had no role in study design, data collection and analysis, decision to publish, or preparation of the manuscript.

\section{Statement of Competing Financial Interests}

All authors declared no competing financial interests.

\section{Disclosure Statement}

All authors declared no conflict of interest related to this work.

\section{References}

1 Rossing P, de Zeeuw D: Need for better diabetes treatment for improved renal outcome. Kidney Int Suppl 2011;120:S28-S32.

$\checkmark 2$ Rosolowsky ET, Skupien J, Smiles AM, Niewczas M, Roshan B, Stanton R, Eckfeldt JH, Warram JH, Krolewski AS: Risk for ESRD in type 1 diabetes remains high despite renoprotection. J Am Soc Nephrol 2011;22:545-553.

-3 Hostetter TH: Prevention of end-stage renal disease due to type 2 diabetes. $\mathrm{N}$ Engl J Med 2001;345:910-912.
-4 Rivero A, Mora C, Muros M, Garcia J, Herrera H, Navarro-Gonzílez JF: Pathogenic perspectives for the role of inflammation in diabetic nephropathy. Clin Sci (Lond) 2009;116: 479-492.

5 Navarro-Gonzalez JF, Mora-Fernandez C: The Role of inflammatory cytokines in diabetic nephropathy. J Am Soc Nephrol 2008; 19:433-442.

-6 Mohamed R, Jayakumar C, Ranganathan PV, Ganapathy V, Ramesh G: Kidney proximal tubular epithelial-specific overexpression of netrin-1 suppresses inflammation and albuminuria through suppression of COX-2-mediated PGE2 production in streptozotocininduced diabetic mice. Am J of Pathol 2012; 181:1991-2002.

7 Ly NP, Komatsuzaki K, Fraser IP, Tseng AA, Prodhan P, Moore KJ, Kinane TB: Netrin-1 inhibits leukocyte migration in vitro and in vivo. Proc Natl Acad Sci U S A 2005; 102: 14729-14734. 
D8 Wang W, Reeves WB, Ramesh G: Netrin-1 and kidney injury. I. Netrin-1 protects against ischemia-reperfusion injury of the kidney. Am J Physiol Renal Physiol 2008;294:F739F747.

-9 Barallobre MJ, Pascual M, Del Rio JA, Soriano E: The Netrin family of guidance factors: emphasis on Netrin-1 signalling. Brain Res Brain Res Rev 2005;49:22-47.

10 Tadagavadi RK, Wang W, Ramesh G: Netrin-1 regulates Th1/Th2/Th17 cytokine production and inflammation through UNC5B receptor and protects kidney against ischemia-reperfusion injury. J Immunol 2010 185:3750-3758.

11 Ranganathan P, Jayakumar C, Navankasattusas S, Li DY, Kim IM, Ramesh G: UNC5B receptor deletion exacerbates tissue injury in response to AKI. J Am Soc Nephrol 2014;25: 239-249.

12 Wang W, Ramesh G: Segment-specific expression of netrin-1 receptors in normal and ischemic mouse kidney. Am J Nephrol 2009; 30:186-193.

13 Tak E, Ridyard D, Badulak A, Giebler A, Shabeka U, Werner T, Clambey E, Moldovan R, Zimmerman MA, Eltzschig HK, Grenz A: Protective role for netrin-1 during diabetic nephropathy. J Mol Med (Berl) 2013;91: 1071-1080.

-14 Navankasattusas S, Whitehead KJ, Suli A, Sorensen LK, Lim AH, Zhao J, Park KW, Wythe JD, Thomas KR, Chien CB, Li DY: The netrin receptor UNC5B promotes angiogenesis in specific vascular beds. Development 2008; 135:659-667.
15 Wilson BD, Ii M, Park KW, Suli A, Sorensen LK, Larrieu-Lahargue F, Urness LD, Suh W, Asai J, Kock GA, Thorne T, Silver M, Thomas KR, Chien CB, Losordo DW, Li DY: Netrins promote developmental and therapeutic angiogenesis. Science 2006;313:640-644

16 Iwano M, Plieth D, Danoff TM, Xue C, Okada H, Neilson EG: Evidence that fibroblasts derive from epithelium during tissue fibrosis. J Clin Invest 2002;110:341-350.

17 Morris SM Jr, Gao T, Cooper TK, Kepka-Lenhart D, Awad AS: Arginase-2 mediates diabetic renal injury. Diabetes 2011;60:30153022.

18 Breyer MD, Böttinger E, Brosius FC 3rd, Coffman TM, Harris RC, Heilig CW, Sharma K; AMDCC: Mouse models of diabetic nephropathy. J Am Soc Nephrol 2005;16:27-45.

19 Brosius FC 3rd, Alpers CE, Bottinger EP, Breyer MD, Coffman TM, Gurley SB, Harris RC, Kakoki M, Kretzler M, Leiter EH, Levi M, McIndoe RA, Sharma K, Smithies O, Susztak K, Takahashi N, Takahashi T; Animal models of diabetic complications consortium: mouse models of diabetic nephropathy. J Am Soc Nephrol 2009;20:2503-2512.

20 Mazelin L, Bernet A, Bonod-Bidaud C, Pays L, Arnaud S, Gespach C, Bredesen DE, Scoazec JY, Mehlen P: Netrin-1 controls colorectal tumorigenesis by regulating apoptosis. $\mathrm{Na}$ ture 2004;431:80-84.

21 Zhang H, Schin M, Saha J, Burke K, Holzman LB, Filipiak W, Saunders T, Xiang M, Heilig CW, Brosius FC 3rd: Podocyte-specific overexpression of GLUT1 surprisingly reduces mesangial matrix expansion in diabetic nephropathy in mice. Am J Physiol Renal Physiol 2010;299:F91-F98.
22 Mohamed R, Ranganathan P, Jayakumar C, Nauta FL, Gansevoort RT, Weintraub NL, Brands M, Ramesh G: Urinary semaphorin $3 \mathrm{~A}$ correlates with diabetic proteinuria and mediates diabetic nephropathy and associated inflammation in mice. J Mol Med (Berl) 2014;92:1245-1256.

23 Wang W, Reeves WB, Pays L, Mehlen P, Ramesh G: Netrin-1 overexpression protects kidney from ischemia reperfusion injury by suppressing apoptosis. Am J Pathol 2009;175: 1010-1018.

24 Rosenberger P, Schwab JM, Mirakaj V, Masekowsky E, Mager A, Morote-Garcia JC, Unertl K, Eltzschig HK: Hypoxia-inducible factor-dependent induction of netrin-1 dampens inflammation caused by hypoxia. Nat Immunol 2009;10:195-202.

25 Mirakaj V, Thix CA, Laucher S, Mielke C, Morote-Garcia JC, Schmit MA, Henes J, Unertl KE, Köhler D, Rosenberger P: Netrin-1 dampens pulmonary inflammation during acute lung injury. Am J Respir Crit Care Med 2010;181:815-824.

26 Aherne CM, Collins CB, Masterson JC, Tizzano M, Boyle TA, Westrich JA, Parnes JA, Furuta GT, Rivera-Nieves J, Eltzschig HK: Neuronal guidance molecule netrin-1 attenuates inflammatory cell trafficking during acute experimental colitis. Gut 2012;61:695-705.

27 Breyer MD, Coffman TM, Flessner MF, Fried LF, Harris RC, Ketchum CJ, Kretzler M, Nelson RG, Sedor JR, Susztak K; Kidney Research National Dialogue (KRND): Diabetic nephropathy: a national dialogue. Clin J Am Soc Nephrol 2013;8:1603-1605. 